Document downloaded from:

http://hdl.handle.net/10251/52620

This paper must be cited as:

Malik, SB.; Thome, N. (2014). On a new generalized inverse for matrices of an arbitrary index. Applied Mathematics and Computation. 226:575-580.

doi:10.1016/j.amc.2013.10.060.

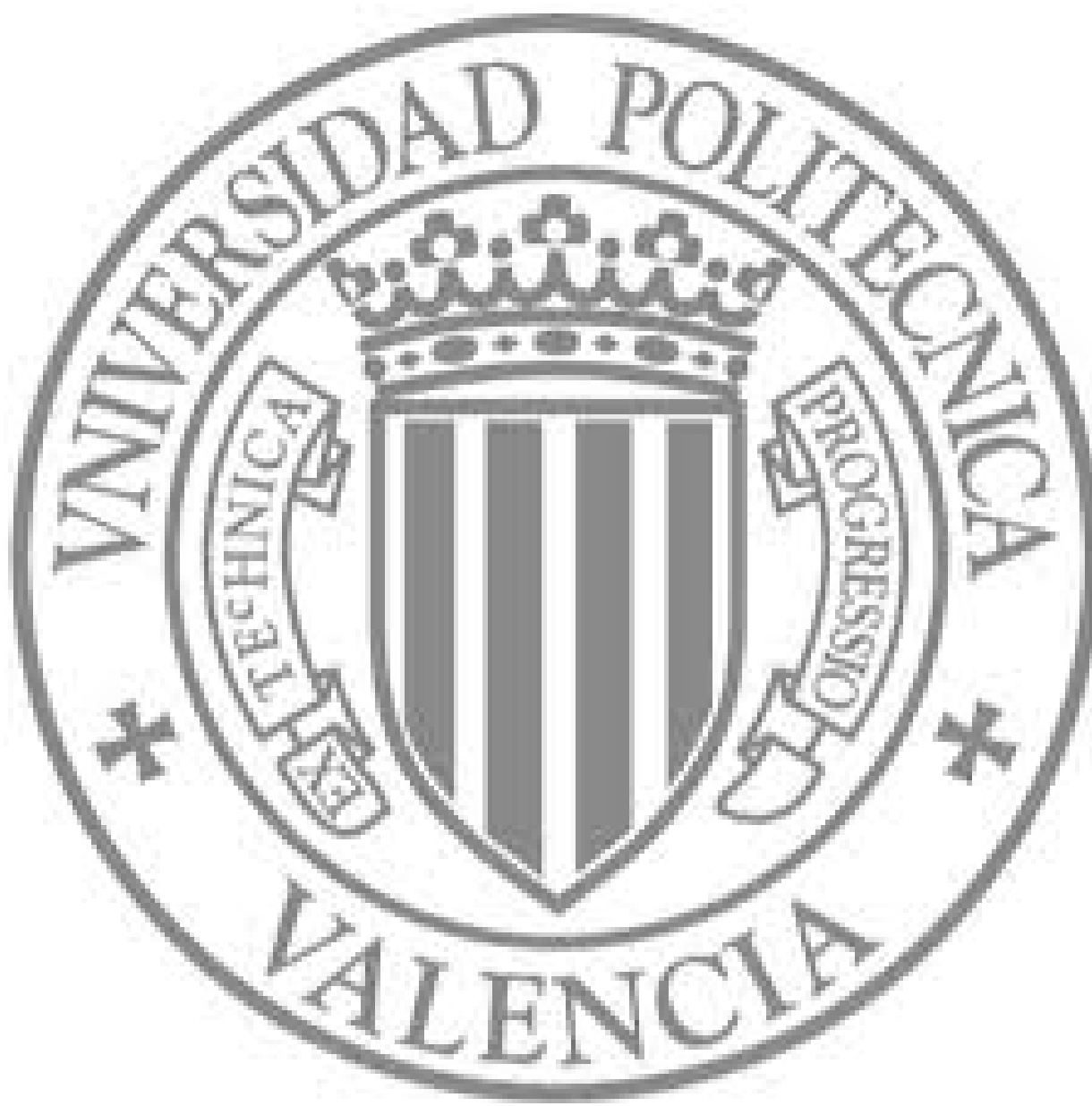

The final publication is available at

http://dx.doi.org/10.1016/j.amc.2013.10.060

Copyright Elsevier 


\title{
On a new generalized inverse for matrices of an arbitrary index
}

\author{
Saroj B. Malik* Néstor Thome ${ }^{\dagger}$
}

\begin{abstract}
The purpose of this paper is to introduce a new generalized inverse, called DMP inverse, associated with a square complex matrix using its Drazin and Moore-Penrose inverses. DMP inverse extends the notion of core inverse, introduced by O.M. Baksalary and G. Trenkler for matrices of index at most 1 in [Core inverse of matrices, Linear and Multilinear Algebra, 2010, 681-697] to matrices of an arbitrary index. DMP inverses are analyzed from both algebraic as well as geometrical approaches establishing the equivalence between them.
\end{abstract}

AMS Classification: 15A09; 15A24

Keywords: Moore-Penrose inverse, Drazin inverse, index, core inverse.

\section{Introduction and background}

The symbol $\mathbb{C}^{m \times n}$ stands for the set of $m \times n$ complex matrices. The symbols $A^{*}, \mathcal{C}(A)$ and $\mathcal{N}(A)$ will denote the conjugate transpose, column space and null space of a matrix $A \in \mathbb{C}^{m \times n}$, respectively. Moreover, $I_{n}$ will refer to the identity matrix of order $n$. If $S$ and $T$ are two complementary subspaces in $\mathbb{C}^{n \times 1}$ (that is, if $\mathbb{C}^{n \times 1}$ is direct sum of $S$ and $T$ ) then the oblique projector onto $S$ along $T$ will be indicated by $P_{S, T}$. For a given matrix $A \in \mathbb{C}^{n \times n}$,

${ }^{*}$ School of Liberal Studies, Ambedkar University, Kashmere Gate, Delhi, India E-mail: saroj.malik@gmail.com .

${ }^{\dagger}$ Instituto Universitario de Matemática Multidisciplinar, Universitat Politècnica de València, 46022, València, Spain. E-mail: njthome@mat.upv.es. This author was partially supported by Ministry of Education of Spain (grant DGI MTM2010-18228). 
this notation will be reduced to $P_{A}$ when $S=\mathcal{C}(A)$ and $T$ is the subspace orthogonal to $S$.

Let $A \in \mathbb{C}^{m \times n}$. The symbol $A^{\dagger}$ stands for the Moore-Penrose inverse of $A$, i.e., the unique matrix satisfying the following four Penrose conditions: $A A^{\dagger} A=A, A^{\dagger} A A^{\dagger}=A^{\dagger}, A A^{\dagger}=\left(A A^{\dagger}\right)^{*}, A^{\dagger} A=\left(A^{\dagger} A\right)^{*}$. A matrix $X$ that satisfies the equality $A X A=A$ is called a $g$-inverse of $A$ and if $X$ satisfies $X A X=X$ it is called an outer inverse of $A$.

For a given matrix $A \in \mathbb{C}^{n \times n}$, recall that the smallest nonnegative integer $m$ such that $\operatorname{rank}\left(A^{m}\right)=\operatorname{rank}\left(A^{m+1}\right)$ is called the index of $A$ and is denoted by $\operatorname{ind}(A)$. The Drazin inverse of $A \in \mathbb{C}^{n \times n}$ is the unique matrix $A^{d} \in \mathbb{C}^{n \times n}$ such that $A^{d} A A^{d}=A^{d}, A A^{d}=A^{d} A, A^{m+1} A^{d}=A^{m}$, where $m=\operatorname{ind}(A)$. If $A \in \mathbb{C}^{n \times n}$ satisfies $\operatorname{ind}(A) \leq 1$, then the Drazin inverse of $A$ is called the group inverse of $A$ and is denoted by $A^{\sharp}$. When it exists, $A^{\sharp}$ is characterized as the unique matrix satisfying the conditions: $A A^{\sharp} A=A, A^{\sharp} A A^{\sharp}=A^{\sharp}$, $A A^{\sharp}=A^{\sharp} A$. For a given matrix $A \in \mathbb{C}^{n \times n}$, the symbol ${ }^{d} C_{A}$ denotes its core-part, that is, ${ }^{d} C_{A}=A A^{d} A$ [4]. The core-part of a matrix $A$ does not reveal any interesting information when $\operatorname{ind}(A) \leq 1$ because in this case it coincides with $A$.

We also recall that a square matrix $A$ is called $E P$ if $A A^{\dagger}=A^{\dagger} A$. Clearly, $A$ is $E P$ if and only if $A^{\sharp}=A^{\dagger}$, that is $E P$ matrices have index at most 1 [5].

The core inverse of a matrix $A \in \mathbb{C}^{n \times n}$ is the unique matrix $A^{\Theta}$ such that $A A^{\Theta}=P_{A}$ and $\mathcal{C}\left(A^{\Theta}\right) \subseteq \mathcal{C}(A)[2,9]$.

While the Moore-Penrose and Drazin inverses of a matrix always exist, the group inverse as well as the core inverse of a square matrix $A$ exist if and only if $A$ and $A^{2}$ have the same rank, i.e., $A$ is of index at most 1 .

Some properties for all these generalized inverses can be found in $[2,3$, $4,7,8]$. All of these generalized inverses are known to be used in important applications. For example, the Moore-Penrose inverse is used to solve the least-squares problem, the group inverse has applications in Markov chain theory, the Drazin inverse gives the solution of a singular linear control system and the core inverse has applications in partial order theory (see for example $[2,3,4,6])$. As the core inverse is the unique least squares g-inverse, it may also be used in some least-squares problems and since the core inverse is also a $\rho$-inverse, it can be used in solving some difference equations.

The aim of this paper is to introduce and analyze a new generalized inverse, namely the DMP inverse, for a square matrix $A$ of an arbitrary index $m$ using the Drazin $A^{d}$ and the Moore-Penrose $A^{\dagger}$ inverses of $A$. First, a 
canonical form for the DMP inverse is established and we conclude that our generalized inverse extends the core inverse introduced in 2010 by O.M. Baksalary and G. Trenkler in [2] in the sense that, while they necessarily require $m \leq 1$, in our case is not necessary. Furthermore, the equivalence of the algebraic definition (Definition 2.3) and the geometrical approach (Theorem 2.13) has been stated. We finally give some of the properties that this new generalized inverse possesses.

\section{A new generalized inverse}

We start this section by defining a new generalized inverse of a square matrix of an arbitrary index. In order to do this, we use the Drazin inverse (D) and the Moore-Penrose (MP) inverse and therefore we name this new generalized inverse as DMP inverse.

Let $A \in \mathbb{C}^{n \times n}$ have index $m$ and consider the system of equations

$$
X A X=X, \quad X A=A^{d} A, \quad A^{m} X=A^{m} A^{\dagger} .
$$

Theorem 2.1. If system (1) has a solution then it is unique.

Proof. Assume that $X_{1}$ and $X_{2}$ satisfy (1), that is $X_{1} A X_{1}=X_{1}, X_{1} A=A^{d} A$, $A^{m} X_{1}=A^{m} A^{\dagger}, X_{2} A X_{2}=X_{2}, X_{2} A=A^{d} A$ and $A^{m} X_{2}=A^{m} A^{\dagger}$. Then, using that $A^{d} A$ is a projector and $A A^{d}=A^{d} A$ we get

$$
\begin{aligned}
X_{1} & =X_{1} A X_{1}=A^{d} A X_{1}=\left(A^{d} A\right)^{m} X_{1}=\left(A^{d}\right)^{m} A^{m} X_{1}=\left(A^{d}\right)^{m} A^{m} A^{\dagger} \\
& =\left(A^{d}\right)^{m} A^{m} X_{2}=\left(A^{d} A\right)^{m} X_{2}=A^{d} A X_{2}=X_{2} A X_{2}=X_{2} .
\end{aligned}
$$

Theorem 2.2. The system of equations (1) is consistent and has a unique solution: $X=A^{d} A A^{\dagger}$.

Proof. It is easy to see that $A^{d} A A^{\dagger}$ satisfies the three equations in system (1). Now, Theorem 2.1 gives the uniqueness.

Thus, for a given square matrix $A$, the matrix $A^{d} A A^{\dagger}$ is the unique matrix satisfying system of equations (1).

Definition 2.3. Let $A \in \mathbb{C}^{n \times n}$ be a matrix of index $m$ (not necessarily $\leq 1$ ). The DMP inverse of $A$, denoted by $A^{d, \dagger}$, is defined to be the matrix

$$
A^{d, \dagger}=A^{d} A A^{\dagger} .
$$


Remark 2.4. Note that the new generalized inverse $A^{d, \dagger}$ can be firstly seen as an extension of that introduced in [9, p. 97] for matrices of index $m \leq 1$. In Remark 2.9 we will present $A^{d, \dagger}$ as an extension of the core inverse.

We now give the canonical form for the DMP inverse of a square matrix $A$ using the Hartwig-Spindelböck decomposition $[5,1]$. For any matrix $A \in$ $\mathbb{C}^{n \times n}$ of rank $r>0$ the Hartwig-Spindelböck decomposition is given by

$$
A=U\left(\begin{array}{cc}
\Sigma K & \Sigma L \\
0 & 0
\end{array}\right) U^{*}
$$

where $U \in \mathbb{C}^{n \times n}$ is unitary, $\Sigma=\operatorname{diag}\left(\sigma_{1} I_{r_{1}}, \sigma_{2} I_{r_{2}}, \ldots, \sigma_{t} I_{r_{t}}\right)$ is a diagonal matrix, the diagonal entries $\sigma_{i}$ being singular values of $A, \sigma_{1}>\sigma_{2}>\ldots>$ $\sigma_{t}>0, r_{1}+r_{2}+\ldots+r_{t}=r$ and $K \in \mathbb{C}^{r \times r}, L \in \mathbb{C}^{r \times(n-r)}$ satisfy $K K^{*}+L L^{*}=$ $I_{r}$. Using this fact we compute the Drazin inverse of $A$ of index $m$ as follows: Let

$$
X=U\left(\begin{array}{ll}
X_{1} & X_{2} \\
X_{3} & X_{4}
\end{array}\right) U^{*}
$$

be the Drazin inverse of $A$ partitioned conformable to $A$. Then $X$ satisfies $X A X=X, A X=X A$ and $A^{m+1} X=A^{m}$. The equation $X A X=X$ implies

$$
\begin{aligned}
& X_{1} \Sigma K X_{1}+X_{1} \Sigma L X_{3}=X_{1}, \\
& X_{3} \Sigma K X_{1}+X_{3} \Sigma L X_{3}=X_{3}, \\
& X_{1} \Sigma K X_{2}+X_{1} \Sigma L X_{4}=X_{2}, \\
& X_{3} \Sigma K X_{2}+X_{3} \Sigma L X_{4}=X_{4}
\end{aligned}
$$

From $A X=X A$ we have

$$
\begin{aligned}
X_{1} \Sigma K & =\Sigma K X_{1}+\Sigma L X_{3}, \\
X_{1} \Sigma L & =\Sigma K X_{2}+\Sigma L X_{4}, \\
X_{3} \Sigma K & =0 \\
X_{3} \Sigma L & =0,
\end{aligned}
$$

and the equality $A^{m+1} X=A^{m}$ implies

$$
\begin{aligned}
& (\Sigma K)^{m+1} X_{1}+(\Sigma K)^{m} \Sigma L X_{3}=(\Sigma K)^{m} \\
& (\Sigma K)^{m+1} X_{2}+(\Sigma K)^{m} \Sigma L X_{4}=(\Sigma K)^{m-1} \Sigma L .
\end{aligned}
$$


Using equations (4)-(13), we have $X_{3}=0$ and $X_{4}=0$. Also, $X_{1} \Sigma K X_{1}=$ $X_{1}, X_{1} \Sigma K X_{2}=X_{2}, X_{1} \Sigma K=\Sigma K X_{1}, X_{1} \Sigma L=\Sigma K X_{2}$ and

$$
\begin{aligned}
& (\Sigma K)^{m+1} X_{1}=(\Sigma K)^{m}, \\
& (\Sigma K)^{m+1} X_{2}=(\Sigma K)^{m-1} \Sigma L .
\end{aligned}
$$

These equations show that the Drazin inverse of $A$ is the matrix

$$
A^{d}=U\left(\begin{array}{cc}
(\Sigma K)^{d} & \left((\Sigma K)^{d}\right)^{2} \Sigma L \\
0 & 0
\end{array}\right) U^{*} .
$$

Also, the Moore-Penrose inverse of $A$ is given as [2]

$$
A^{\dagger}=U\left(\begin{array}{cc}
K^{*} \Sigma^{-1} & 0 \\
L^{*} \Sigma^{-1} & 0
\end{array}\right) U^{*} .
$$

Now, the DMP inverse of $A$ is

$$
\begin{aligned}
A^{d, \dagger} & =A^{d} A A^{\dagger} \\
& =U\left(\begin{array}{cc}
(\Sigma K)^{d} & \left((\Sigma K)^{d}\right)^{2} \Sigma L \\
0 & 0
\end{array}\right)\left(\begin{array}{cc}
\Sigma K & \Sigma L \\
0 & 0
\end{array}\right)\left(\begin{array}{cc}
K^{*} \Sigma^{-1} & 0 \\
L^{*} \Sigma^{-1} & 0
\end{array}\right) U^{*} \\
& =U\left(\begin{array}{cc}
(\Sigma K)^{d} & 0 \\
0 & 0
\end{array}\right) U^{*} .
\end{aligned}
$$

We can summarize this reasoning in the following result.

Theorem 2.5. Let $A \in \mathbb{C}^{n \times n}$ be of the form (2). Then

$$
A^{d, \dagger}=U\left(\begin{array}{cc}
(\Sigma K)^{d} & 0 \\
0 & 0
\end{array}\right) U^{*} .
$$

Remark 2.6. Notice that in (14) we have provided a representation for the Drazin inverse of a square matrix $A$. If we denote

$$
C_{m}=(\Sigma K)^{m-1} \Sigma\left((\Sigma K)^{2 m} \Sigma\right)^{\dagger}(\Sigma K)^{m-1} \Sigma,
$$

a well-known similar representation for $A^{d}$ (see $[1$, p. 2803]) is

$$
A^{d}=U\left(\begin{array}{cc}
C_{m} K & C_{m} L \\
0 & 0
\end{array}\right) U^{*} .
$$

We remark that this last formula uses the Moore-Penrose inverse of $(\Sigma K)^{2 m} \Sigma$ while the representation (14) involves the Drazin inverse of $\Sigma K$. 
Remark 2.7. We can observe that $(\Sigma K)^{d}$ is $p(\Sigma K)$ for some polynomial $p(x)$ (see Theorem 7.5.1 in [4] and Theorem 2.4.30 in [6]). When $K$ is singular, 0 is a root of $p$. Consequently, $A^{d, \dagger}$ is the polynomial matrix

$$
A^{d, \dagger}=U p\left(\left(\begin{array}{cc}
\Sigma K & 0 \\
0 & 0
\end{array}\right)\right) U^{*}=p\left(U\left(\begin{array}{cc}
\Sigma K & 0 \\
0 & 0
\end{array}\right) U^{*}\right) .
$$

Lemma 2.8. Let $A \in \mathbb{C}^{n \times n}$ be a matrix of index $m$ written as in (2). Then $\operatorname{ind}(\Sigma K)=m-1$.

Proof. Since

$$
A^{m}=U\left(\begin{array}{cc}
(\Sigma K)^{m-1} & O \\
O & O
\end{array}\right)\left(\begin{array}{cc}
\Sigma K & \Sigma L \\
Y & Z
\end{array}\right) U^{*}
$$

for some $Y, Z$ of adequate sizes such that the matrix $\left(\begin{array}{cc}\Sigma K & \Sigma L \\ Y & Z\end{array}\right)$ is nonsingular, we have $\operatorname{rank}\left(A^{m}\right)=\operatorname{rank}\left((\Sigma K)^{m-1}\right)$. Similarly, $\operatorname{rank}\left(A^{m+1}\right)=$ $\operatorname{rank}\left((\Sigma K)^{m}\right)$. From $\operatorname{ind}(A)=m$ we get that $m-1$ is the smallest nonnegative integer such that $\operatorname{rank}\left((\Sigma K)^{m-1}\right)=\operatorname{rank}\left((\Sigma K)^{m}\right)$, that is $\operatorname{ind}(\Sigma K)=$ $m-1$.

Remark 2.9. Notice that if $A$ has index 1, the generalized inverse in Definition 2.3 coincides with the core inverse of $A$ introduced and studied by O.M. Baksalary and G. Trenkler in [2].

There is another (outer) inverse associated with a square matrix $A$, namely $A^{\dagger, d}=A^{\dagger} A A^{d}$ and its canonical form in terms of the Hartwig-Spindelböck decomposition of $A$ is given by

$$
A^{\dagger, d}=U\left(\begin{array}{cc}
\left(K^{*} K\right)(\Sigma K)^{d} & \left(K^{*} K\right)\left((\Sigma K)^{d}\right)^{2} \Sigma L \\
\left(L^{*} K\right)(\Sigma K)^{d} & \left(L^{*} K\right)\left((\Sigma K)^{d}\right)^{2} \Sigma L
\end{array}\right) U^{*} .
$$

It is expected that this will have properties similar to that of $A^{d, \dagger}$. The following example shows that in general both inverses $A^{\dagger, d}$ and $A^{d, \dagger}$ are different.

Example 2.10. If $A=\left(\begin{array}{ll}1 & 1 \\ 0 & 0\end{array}\right)$ then simple computations give

$$
A^{d, \dagger}=\left(\begin{array}{ll}
1 & 0 \\
0 & 0
\end{array}\right) \text { and } A^{\dagger, d}=\left(\begin{array}{cc}
1 / 2 & 1 / 2 \\
1 / 2 & 1 / 2
\end{array}\right)
$$


We know that if $A^{d}=A^{\dagger}$ then $\operatorname{ind}(A) \leq 1$. In such a case, clearly, $A^{\dagger, d}=$ $A^{d, \dagger}=A^{\dagger}=A^{\sharp}$. Moreover, if $A^{d}=A$ then $A^{d, \dagger}=A^{2} A^{\dagger}$ and $A^{\dagger, d}=A^{\dagger} A^{2}$. If $A$ is an EP tripotent matrix then $A^{\dagger}=A$ and so $A^{d, \dagger}=A^{2} A^{d}={ }^{d} C_{A}=A^{\dagger, d}$. It may be worth noting that $A^{\dagger, d}$ and $A^{d, \dagger}$ have always the same core-part since $A A^{d, \dagger} A=A A^{d} A={ }^{d} C_{A}, A A^{\dagger, d} A=A A^{d} A={ }^{d} C_{A}$.

In what follows, we shall confine to studying the DMP inverse. The next result gives some properties of the DMP inverse.

Theorem 2.11. The DMP inverse $A^{d, \dagger}$ of a square matrix $A$ satisfies the equations (i) $A X A={ }^{d} C_{A}$, and (ii) $A X={ }^{d, \dagger} C_{A} A^{\dagger}$ (in the unknown $X$ ), where ${ }^{d, \dagger} C_{A}=A A^{d, \dagger} A$ denotes the DMP core-part of $A$.

Proof. (i) Using the Hartwig-Spindelböck decomposition of $A$, simple computations show that ${ }^{d} C_{A}=A A^{d} A$ and ${ }^{d, \dagger} C_{A}=A A^{d, \dagger} A$ are given by

$$
{ }^{d} C_{A}=U\left(\begin{array}{cc}
{ }^{d} C_{\Sigma K} & \Sigma K(\Sigma K)^{d} \Sigma L \\
0 & 0
\end{array}\right) U^{*}
$$

and

$$
{ }^{d, \dagger} C_{A}=U\left(\begin{array}{cc}
{ }^{d} C_{\Sigma K} & \Sigma K(\Sigma K)^{d} \Sigma L \\
0 & 0
\end{array}\right) U^{*}
$$

Therefore, ${ }^{d} C_{A}={ }^{d, \dagger} C_{A}$, i.e., the usual core-part of $A$ is its DMP core-part. So, $A^{d, \dagger}$ is a solution of $A X A={ }^{d} C_{A}$.

(ii) is easy to prove.

Theorem 2.12. If $A \in \mathbb{C}^{n \times n}$ has index $m$ then the following statements hold:

(a) $A A^{d, \dagger}$ is a projector onto $\mathcal{C}\left({ }^{d} C_{A}\right)$ along $\mathcal{N}\left(A^{d} A^{\dagger}\right)$.

(b) $A^{d, \dagger} A=A^{d} A$ is a projector onto $\mathcal{C}\left(A^{m}\right)$ along $\mathcal{N}\left(A^{m}\right)$.

Proof. It is clear that $A A^{d, \dagger}$ is a projector because $A^{d, \dagger}$ is an outer inverse of $A$. Moreover, $\mathcal{C}\left(A A^{d, \dagger}\right)=A A^{d} \mathcal{C}\left(A A^{\dagger}\right)=A A^{d} \mathcal{C}(A)=\mathcal{C}\left(A A^{d} A\right)=\mathcal{C}\left({ }^{d} C_{A}\right)$ and $\mathcal{N}\left(A A^{d, \dagger}\right)=\mathcal{N}\left(A A^{d} A A^{\dagger}\right)=\mathcal{N}\left(A^{d} A A^{\dagger}\right)=\mathcal{N}\left(A^{d} A^{\dagger}\right)$.

Item (b) can be deduced from the properties of the projector $A^{d} A[4]$.

In Definition 2.3 the DMP generalized inverse has been introduced from an algebraic approach. Next result presents a characterization of the DMP inverse from a geometrical point of view. 
Theorem 2.13. If $A \in \mathbb{C}^{n \times n}$ has index $m$ then $A^{d, \dagger}$ is the unique matrix $X$ that satisfies

$$
A X=P_{\mathcal{C}\left({ }^{d} C_{A}\right), \mathcal{N}\left(A^{d} A^{\dagger}\right)}, \mathcal{C}(X) \subseteq \mathcal{C}\left(A^{m}\right) .
$$

Proof. The fact that $A A^{d, \dagger}$ is idempotent follows from Theorem 2.12. Moreover, $\mathcal{C}\left(A^{d, \dagger}\right)=\mathcal{C}\left(A^{d} A A^{\dagger}\right) \subseteq \mathcal{C}\left(A^{d} A\right)=\mathcal{C}\left(A^{m}\right)$. It remains to prove that there is only one $X$ satisfying conditions (16).

Suppose that $X_{1}, X_{2}$ satisfy (16). Then $A X_{1}=A X_{2}=P_{\mathcal{C}\left({ }^{d} C_{A}\right), \mathcal{N}\left(A^{d} A^{\dagger}\right)}$, $\mathcal{C}\left(X_{1}\right) \subseteq \mathcal{C}\left(A^{m}\right)$ and $\mathcal{C}\left(X_{2}\right) \subseteq \mathcal{C}\left(A^{m}\right)$. Since $A\left(X_{1}-X_{2}\right)=0$, we get $\mathcal{C}\left(X_{1}-\right.$ $\left.X_{2}\right) \subseteq \mathcal{N}(A)$. From $\mathcal{C}\left(X_{1}\right) \subseteq \mathcal{C}\left(A^{m}\right)$ and $\mathcal{C}\left(X_{2}\right) \subseteq \mathcal{C}\left(A^{m}\right)$ we get $\mathcal{C}\left(X_{1}-X_{2}\right) \subseteq$ $\mathcal{C}\left(A^{m}\right)$, that is $\mathcal{C}\left(X_{1}-X_{2}\right) \subseteq \mathcal{N}\left(A^{m}\right) \cap \mathcal{C}\left(A^{m}\right)=\{0\}$ since $A$ has index $m$. Thus, $X_{1}=X_{2}$.

Note that, if $A$ has index at most 1 then $P_{\mathcal{C}\left({ }^{d} C_{A}\right), \mathcal{N}\left(A^{d} A^{\dagger}\right)}=P_{\mathcal{C}(A), \mathcal{N}\left(A^{*}\right)}[2]$.

We can conclude that $A^{d, \dagger}$ is the only matrix that satisfies equations (1) and also $A^{d, \dagger}$ is the only matrix that satisfies relations (16). So, both algebraic and geometrical approaches are equivalent.

\subsection{Some properties and remarks}

DMP inverses provide a new class of generalized inverses because in general the DMP inverse of a matrix is different from each of its Moore-Penrose inverse, the group inverse and the Darzin inverse. In fact, in the Example 2.10 we have shown a matrix $A$ of index 1 such that $A^{\#}, A^{\dagger}$ and $A^{d, \dagger}$ are all different. As another example (when the matrix involved is of index greater than 1) we can consider the matrix

$$
B=\left(\begin{array}{llll}
1 & 1 & 0 & 0 \\
0 & 0 & 0 & 0 \\
0 & 0 & 0 & 1 \\
0 & 0 & 0 & 0
\end{array}\right)
$$

It is easy to check that $\operatorname{ind}(B)=2$ and

$$
B^{d}=\left(\begin{array}{cccc}
1 & 1 & 0 & 0 \\
0 & 0 & 0 & 0 \\
0 & 0 & 0 & 0 \\
0 & 0 & 0 & 0
\end{array}\right), B^{\dagger}=\left(\begin{array}{cccc}
1 / 2 & 0 & 0 & 0 \\
1 / 2 & 0 & 0 & 0 \\
0 & 0 & 0 & 0 \\
0 & 0 & 1 & 0
\end{array}\right), B^{d, \dagger}=\left(\begin{array}{cccc}
1 & 0 & 0 & 0 \\
0 & 0 & 0 & 0 \\
0 & 0 & 0 & 0 \\
0 & 0 & 0 & 0
\end{array}\right) .
$$


The matrix $B$ also allows us to confirm that, in general, the inverse $B^{d, \dagger}$ is not a $g$-inverse of $B$.

Next results state some properties that the DMP inverse inherits from the core inverse.

Proposition 2.14. Let $A \in \mathbb{C}^{n \times n}$ be a matrix of index $m$. Then

(a) $A^{d, \dagger}=A^{d} P_{A}$.

(b) $A^{d, \dagger}$ is an outer inverse of $A$.

(c) $\left(A^{d, \dagger}\right)^{n}=\left\{\begin{array}{cl}\left(A^{d} A^{\dagger}\right)^{n / 2} & \text { if } n \text { is even } \\ A\left(A^{d} A^{\dagger}\right)^{(n+1) / 2} & \text { if } n \text { is odd }\end{array}\right.$.

(d) $\left(A^{d, \dagger}\right)^{\dagger}=\left(\left(A P_{A}\right)^{d}\right)^{\dagger}$.

(e) $\left(\left(A^{d, \dagger}\right)^{d}\right)^{d}=A^{d, \dagger}$.

(f) $A A^{d, \dagger}=A^{d, \dagger} A$ if and only if $\mathcal{N}\left(A^{\dagger}\right) \subseteq \mathcal{N}\left(A^{d}\right)$.

(g) $A^{d, \dagger}=0$ if and only if $A$ is nilpotent or $A=0$.

(h) $A^{d, \dagger}=A$ if and only if $A$ is EP and tripotent.

Proof. (a) and (b) follow from definition and properties of the Moore-Penrose and Drazin inverses.

(c) First we compute $\left(A^{d, \dagger}\right)^{1}=A^{d} A A^{\dagger}=A A^{d} A^{\dagger}$ and also $\left(A^{d, \dagger}\right)^{2}=$ $A^{d} A A^{\dagger} A^{d} A A^{\dagger}=A^{d}\left(A A^{\dagger} A\right) A^{d} A^{\dagger}=A^{d} A A^{d} A^{\dagger}=A^{d} A^{\dagger}$. Now, by induction it is easy to obtain the general formula.

(d) and (e) follow as an application of Theorem 2.5.

(f) Since $I-A A^{\dagger}$ and $A^{d} A$ are projectors we have that $A A^{d, \dagger}=A^{d, \dagger} A$ $\Longleftrightarrow A A^{d} A A^{\dagger}=A^{d} A \Longleftrightarrow A A^{d}\left(I-A A^{\dagger}\right)=O \Longleftrightarrow \mathcal{R}\left(I-A A^{\dagger}\right) \subseteq \mathcal{N}\left(A^{d} A\right)$ $\Longleftrightarrow \mathcal{N}\left(A^{\dagger}\right) \subseteq \mathcal{N}\left(A^{d}\right)$.

(g) Assume that $A \neq 0$ and $A^{d, \dagger}=0$. If we write $A$ as in (2) then $A^{d, \dagger}$ can expressed as in Theorem 2.5. Now, we split the proof in two cases:

(i) $\Sigma K \neq 0$. In this case, from $A^{d, \dagger}=0$ we obtain $(\Sigma K)^{d}=0$. So, $\Sigma K$ is nilpotent. Hence, $A$ must be nilpotent.

(ii) $\Sigma K=0$. In this case, the matrix $A=U\left(\begin{array}{cc}0 & \Sigma L \\ 0 & 0\end{array}\right) U^{*}$ is clearly nilpotent. 
The converse is evident because in both $A=0$ and $A$ nilpotent cases its Drazin inverse is the null matrix.

(h) The case $A=0$ is trivial. Assume that $A \neq 0$ and it is represented as in (2). Then $A^{d, \dagger}$ can expressed as in Theorem 2.5. Hence, $A^{d, \dagger}=A \Longleftrightarrow$ $(\Sigma K)^{d}=\Sigma K, \Sigma L=0 \Longleftrightarrow(\Sigma K)^{3}=\Sigma K, L=0 \Longleftrightarrow A^{3}=A, A$ is $E P$, where in the last equivalence we have used Lemma $1(\mathrm{v})$ in [2].

From item (h) in Proposition 2.14 and [2, Theorem 2(v)] we can notice that $A^{d, \dagger} \neq A$ when $\operatorname{ind}(A)>1$.

Proposition 2.15. Let $A \in \mathbb{C}^{n \times n}$ be a (nonzero) matrix of index $m$ written as in (2) and $A^{d, \dagger}$ expressed as in Theorem 2.5. Then

(a) $A^{d, \dagger}$ is EP if and only if $(\Sigma K)^{d}$ is EP.

(b) $\left(A^{d, \dagger}\right)^{\dagger}=U\left(\begin{array}{cc}\left((\Sigma K)^{d}\right)^{\dagger} & 0 \\ 0 & 0\end{array}\right) U^{*}$.

Proof. (a) and (b) follow as an application of Theorem 2.5.

We remark that property (a) in the previous proposition reduces to the fact that $A^{d, \dagger}$ is $E P$ when $\operatorname{ind}(A) \leq 1$.

Finally, it seems to be natural to enquire whether the DMP inverse specifies a matrix partial ordering as the core inverse does (see [2]). This problem will be addressed in a forthcoming paper.

\section{Acknowledgements}

The authors would like to thank the referees for their valuable comments and suggestions, which allowed improving considerably the writing of the paper.

\section{References}

[1] O.M. Baksalary, G.P.H. Styan, G. Trenkler, On a matrix decomposition of Hartwig and Spindelböck. Linear Algebra and its Applications, 430, 2798-2812, 2009.

[2] O.M. Baksalary, G. Trenkler, Core inverse of matrices, Linear and Multilinear Algebra, 58, 6, 681-697, 2010. 
[3] A. Ben-Israel, T. Greville, Generalized Inverses: Theory and Applications. John Wiley \& Sons, Second Edition, 2003.

[4] S.L. Campbell, C.D. Meyer Jr., Generalized Inverse of Linear Transformations. Dover, New York, Second Edition, 1991.

[5] R.E. Hartwig, K. Spindleböck, Matrices for which $A^{*}$ and $A^{\dagger}$ commmute. Linear and Multilinear Algebra, 14, 241-256, 1984.

[6] S.K. Mitra, P. Bhimasankaram, S.B. Malik, Matrix Partial Orders, Shorted Operators and Applications. World Scientific Publishing Company, 2010.

[7] D. Mosić, D.S. Djordjević, New characterizations of EP, generalized normal and generalized Hermitian elements in rings, Applied Mathematics and Computation, 218, 6702-6710, 2012.

[8] P. Patricio, A. Veloso da Costa, On the Drazin index of regular elements, Central European Journal of Mathematics, 7, 2 200-205, 2009.

[9] C.R. Rao, S.K. Mitra, Generalized Inverse of Matrices and its Applications. John Wiley \& Sons, New York, 1971. 